\title{
Strangelets at finite temperature in a baryon density-dependent quark mass model
}

\author{
Huai-Min Chen $\odot,{ }^{1}$ Cheng-Jun Xia ${ }^{2, *}$ and Guang-Xiong Peng $\circledast^{1,3,4, \dagger}$ \\ ${ }^{1}$ School of Nuclear Science and Technology, University of Chinese Academy of Sciences, \\ Beijing 100049, China \\ ${ }^{2}$ Center for Gravitation and Cosmology, College of Physical Science and Technology, \\ Yangzhou University, Yangzhou 225009, China \\ ${ }^{3}$ Theoretical Physics Center for Science Facilities, Institute of High Energy Physics, \\ P.O. Box 918, Beijing 100049, China \\ ${ }^{4}$ Synergetic Innovation Center for Quantum Effects and Application, Hunan Normal University, \\ Changsha 410081, China
}

(Received 20 October 2021; accepted 6 December 2021; published 7 January 2022)

\begin{abstract}
The properties of strangelets at finite temperature are studied within the framework of a baryon densitydependent quark mass model, where a new quark mass scaling and self-consistent thermodynamic treatment are adopted. The effects of finite volume and Coulomb energy are taken into account. Our results show that the temperature $T$, baryon number $A$, and perturbation interactions have strong influences on the properties of strangelets. It is found that the energy per baryon $M / A$ and charge-to-mass ratio $f_{z}$ decrease with baryon number $A$, while the mechanically stable radius $R$ and strangeness per baryon $f_{S}$ are increasing. For a strangelet with a fixed baryon number, we note that as temperature $T$ increases the quantities $M / A, R$, and $f_{S}$ are increasing while $f_{z}$ is decreasing. The effects of confinement and perturbative interactions are investigated as well by readjusting the corresponding parameters.
\end{abstract}

DOI: 10.1103/PhysRevD.105.014011

\section{INTRODUCTION}

Since Bodmer studied the possible collapse of finite nuclei in 1971 [1], the properties of quark matter have begun to attract much attention, which is particularly the case after Witten proposed that strange quark matter (SQM) might be the true ground state of strong interaction in 1984 [2]. Compared with ordinary nuclear matter, the density of quark matter is much larger so that the Fermi energy of $u$ and $d$ quarks can easily surpass the rest mass of $s$ quarks. Then it is energetically favorable to convert $u$ and $d$ quarks into $s$ quarks and form SQM. Farhi and Jaffe then investigated the properteis of SQM based on the MIT bag model and found that the energy per baryon can be lower than that of ${ }^{56} \mathrm{Fe}(930 \mathrm{MeV})$ in a large parameter space; i.e., SQM is absolutely stable [3]. If true, both strange stars and strangelets made of SQM are expected to

\footnotetext{
cjxia@nit.zju.edu.cn

gxpeng@ucas.ac.cn
}

Published by the American Physical Society under the terms of the Creative Commons Attribution 4.0 International license. Further distribution of this work must maintain attribution to the author(s) and the published article's title, journal citation, and DOI. Funded by SCOAP. be observed in the Universe. Extensive studies on SQM were then carried out [4-8].

SQM nuggets with baryon number $A$ less than $10^{7}$ are called strangelets [9] or slets for short [10]. There are mainly two methods to produce strangelets, i.e., by nature or by experiments. For example, it is expected that the collision of strange stars may eject strangelets, which could eventually reach Earth [11-16]. During the hadronization process of the early Universe, strangelets may form and constitute dark matter [17]. The collision of highly energetic cosmic rays with Earth's atmosphere may also produce strangelets [15]. Additionally, it is possible that strangelets are produced in heavy ion collision experiments [18-21].

At present, the MIT bag model has been widely used to study the properties of strangelets and obtained some important results. For example, Farhi and Jaffe found that the strangelets have a small amount of positive charge under weak equilibrium condition [3]. Greiner and coworkers investigated the possibility to synthesize strangelets via heavy ion collisions [22,23]. Adopting the MIT bag model, Madsen considered the finite size effects of strangelets at vanishing temperatures [24-26], while Mustafa and Ansari studied the stability of finite-size strangelets at finite temperatures [27]. The effects of finite temperature on the stability and thermodynamical properties of strangelets 
were later investigated by Mustafa and Ansari [27] and Zhang and $\mathrm{Su}$ [28]. In addition, taking into account the contributions of electrostatic effects, Debye screening, and nonzero surface tensions, Alford et al. had found the critical value of surface tension to maintain the stability of strangelets [29]. Recently, Lugones and Grunfeld found that the surface tension in the MIT bag model would be below the critical value, favoring the existence of a strangelet crust [30]. However, such a conclusion could be significantly modified if vector interactions are considered, which drastically increase the surface and curvature tensions [31].

In addition to the MIT bag model, there are various effective models reflecting QCD characteristics, e.g., perturbation models [32], quark-cluster model [33,34], and so on [4,5,35-42]. The properties of strangelets were thus investigated with those effective models, e.g., NJL model [43] and quasiparticle model [44]. The baryon densitydependent quark mass model has also been used to study the properties of strangelets, and many interesting conclusions have been drawn at $T=0[45,46]$. In this paper, we apply the baryon densitydependent quark mass model to study the properties of strangelets at finite temperature, where the effects of various quark interactions are examined by adopting different parameter sets after we consider the contributions of the Coulomb interaction.

The paper is organized as follows. In Sec. II, we give the thermodynamic treatment of strangelets at finite temperatures in the framework of the baryon densitydependent quark mass model, where the contributions of the Coulomb interaction are accounted for. In Sec. III, we consider the quark mass scaling and gluon mass scaling for SQM at finite temperatures, where both the confinement interaction and perturbation interaction are considered in the equivalent mass of quarks. The relationship between gluon mass and temperature is discussed as well. In Sec. IV, we present the numerical results on the properties of strangelets at finite temperature, where the energy per baryon, radius, and charges of strangelets are discussed. Finally, a summary is given in Sec. V.

\section{SELF-CONSISTENT THERMODYNAMIC TREATMENT}

The most complex and controversial issue in baryon densitydependent quark mass models is thermodynamic self-consistency, where various thermodynamic treatments were developed based on different considerations [5,47-49]. In this paper, we start from the free energy density $F$ and obtain the other thermodynamic quantities through self-consistent thermodynamic treatment, i.e., taking $F$ as the characteristic thermodynamic function. The free energy density is expressed in the same form as the free particle system, but the mass is replaced by the equivalent one which depends on temperature and density. The free energy density is given by

$$
\begin{aligned}
F & =F\left(T, V,\left\{n_{i}\right\},\left\{m_{i}\right\}\right) \\
& =\Omega_{0}\left(T, V,\left\{\mu_{i}^{*}\right\},\left\{m_{i}\right\}\right)+\sum_{i=u, d, s} \mu_{i}^{*} n_{i},
\end{aligned}
$$

where $T$ is the temperature, $n_{i}$ the particle number density, $m_{i}$ the mass, and $\mu_{i}^{*}$ the effective chemical potential of particle type $i$. Note that $\Omega_{0}$ corresponds to the thermodynamic potential density of a system composed of noninteracting particles, which should be viewed as an intermediate variable instead of the real one. The corresponding differential equation is then obtained with

$$
\begin{aligned}
\mathrm{d} F= & \frac{\partial \Omega_{0}}{\partial T} \mathrm{~d} T+\frac{\partial \Omega_{0}}{\partial V} \mathrm{~d} V \\
& +\sum_{i}\left(\frac{\partial \Omega_{0}}{\partial \mu_{i}^{*}} \mathrm{~d} \mu_{i}^{*}+\mu_{i}^{*} \mathrm{~d} n_{i}+n_{i} \mathrm{~d} \mu_{i}^{*}\right) \\
& +\sum_{i} \frac{\partial \Omega_{0}}{\partial m_{i}}\left(\sum_{j} \frac{\partial m_{i}}{\partial n_{j}} \mathrm{~d} n_{j}+\frac{\partial m_{i}}{\partial T} \mathrm{~d} T\right) \\
= & {\left[\frac{\partial \Omega_{0}}{\partial T}+\sum_{i} \frac{\partial \Omega_{0}}{\partial m_{i}} \frac{\partial m_{i}}{\partial T}\right] \mathrm{d} T+\frac{\partial \Omega_{0}}{\partial V} \mathrm{~d} V } \\
& +\sum_{i}\left[\mu_{i}^{*}+\sum_{j} \frac{\partial \Omega_{0}}{\partial m_{j}} \frac{\partial m_{j}}{\partial n_{i}}\right] \mathrm{d} n_{i} .
\end{aligned}
$$

Comparing with the basic thermodynamic differential relation

$\mathrm{d} F=-S \mathrm{~d} T+\left(-P-F+\sum_{i} \mu_{i} n_{i}\right) \frac{\mathrm{d} V}{V}+\sum_{i} \mu_{i} \mathrm{~d} n_{i}$,

we can then obtain entropy density $S$, pressure $P$, and chemical potential $\mu_{i}$ with

$$
\begin{gathered}
S=-\frac{\partial \Omega_{0}}{\partial T}-\sum_{i} \frac{\partial m_{i}}{\partial T} \frac{\partial \Omega_{0}}{\partial m_{i}}, \\
P=-F+\sum_{i} \mu_{i} n_{i}-V \frac{\partial \Omega_{0}}{\partial V} \\
\mu_{i}=\mu_{i}^{*}+\sum_{j} \frac{\partial \Omega_{0}}{\partial m_{j}} \frac{\partial m_{j}}{\partial n_{i}} .
\end{gathered}
$$

The particle number densities $n_{i}=n_{i}^{+}-n_{i}^{-}$and the energy density $E$ are then fixed by

$$
\begin{gathered}
n_{i}^{ \pm}=-\frac{\partial \Omega_{0}^{ \pm}}{\partial \mu_{i}^{*}}, \\
E=F+T S=\Omega_{0}+\sum_{i} \mu_{i}^{*} n_{i}+T S .
\end{gathered}
$$

Because the defined strangelet baryon number $A$ is less than $10^{7}$, its radius is usually smaller than the Compton 
wavelength of the electron, so that electrons are not confined within the strangelets. Then, we can neglect electrons, and the corresponding chemical potential is exactly zero if we minimize the mass of a strangelet. This property has two implications, i.e., the relaxation of the local charge neutrality condition and the contributions of the Coulomb interaction in the weak-equilibrium condition for strangelets. Considering the contribution of volume charge, the interaction energy between volume charge and surface charge, and the contribution of surface charge, we can obtain the Coulomb energy as follows:

$$
\begin{aligned}
\bar{E}_{\mathrm{C}} & =\frac{3 \alpha_{\mathrm{C}} \bar{Q}_{\mathrm{v}}{ }^{2}}{5 R}+e \phi_{\mathrm{v}}(R) \bar{Q}_{\mathrm{s}}+\frac{e \phi_{\mathrm{s}}(R) \bar{Q}_{\mathrm{s}}}{2} \\
& =\frac{\alpha_{\mathrm{C}}}{2 R}\left(\bar{Q}^{2}+\frac{1}{5} \bar{Q}_{\mathrm{v}}{ }^{2}\right)
\end{aligned}
$$

where $e \phi_{\mathrm{v}}(R)=\alpha_{\mathrm{C}} \bar{Q}_{\mathrm{v}} / R, e \phi_{\mathrm{s}}(R)=\alpha_{\mathrm{C}} \bar{Q}_{\mathrm{s}} / R . \bar{E}_{\mathrm{C}}$ is the total energy of the Coulomb, and $\alpha_{C} \approx 1 / 137$ represents the fine structure constant. Then, we have

$$
E_{\mathrm{C}}=\frac{3 \bar{E}_{\mathrm{C}}}{4 \pi R^{3}}=\frac{2}{15} \pi R^{2} \alpha_{\mathrm{C}}\left(5 Q^{2}+Q_{\mathrm{v}}^{2}\right),
$$

where $E_{\mathrm{C}}=\bar{E}_{\mathrm{C}} / V$ is the Coulomb energy density, and $Q_{V}$ represents the volume contribution of total charge density $Q$, namely, $Q=\sum_{i} q_{i} n_{i}$, and $Q_{V}=\sum_{i} q_{i} n_{i, V}$. The charge of each particle is $q_{u}=\frac{2}{3}, q_{d}=-\frac{1}{3}, q_{s}=-\frac{1}{3}$. The total energy density of a strangelet is then obtained with

$$
E_{\text {total }}=E+E_{\mathrm{C}}
$$

where $E$ is fixed by Eq. (8) and $E_{c}$ by Eq. (10).

For the pressure, entropy, and chemical potential, the contribution of the Coulomb energy can be obtained with Eqs. (4)-(6), which are expressed in terms of $P_{\mathrm{C}}, S_{\mathrm{C}}$, and $\mu_{i, \mathrm{C}}$, respectively. According to the basic differential relation of thermodynamics, they satisfy the following differential expression:

$$
\begin{aligned}
\mathrm{d} E_{\mathrm{C}}= & \left(T S_{\mathrm{C}}-P_{\mathrm{C}}-E_{\mathrm{C}}+\sum_{i} \mu_{i, \mathrm{C}} n_{i}\right) \frac{\mathrm{d} V}{V} \\
& +\sum_{i} \mu_{i, \mathrm{C}} \mathrm{d} n_{i}+T \mathrm{~d} S_{\mathrm{C}} .
\end{aligned}
$$

Meanwhile, the Coulomb energy density of a strangelet is a function of the quark number density and volume, i.e., $E_{\mathrm{C}}=E_{\mathrm{C}}\left(V, n_{i}\right)$. This indicates

$$
\mathrm{d} E_{\mathrm{C}}=\sum_{i} \frac{\partial E_{\mathrm{C}}}{\partial n_{i}} d n_{i}+\frac{\partial E_{\mathrm{C}}}{\partial V} \mathrm{~d} V
$$

Then, we have

$$
\begin{gathered}
\mu_{i, \mathrm{C}}=\frac{\partial E_{\mathrm{C}}}{\partial n_{i}}=\frac{\partial E_{\mathrm{C}}}{\partial m_{i}} / \frac{\partial n_{i}}{\partial m_{i}}=\frac{4}{3} \pi R^{2} \alpha_{\mathrm{C}} Q q_{i}, \\
S_{\mathrm{C}}=0, \\
P_{\mathrm{C}}=-E_{\mathrm{C}}+\sum_{i} \mu_{i, \mathrm{C}} n_{i}-V \frac{\partial E_{\mathrm{C}}}{\partial V} \\
=\frac{2}{9} \pi R^{2} \alpha_{\mathrm{C}}\left(Q^{2}-Q_{\mathrm{v}}^{2}\right)-\frac{4}{9} \pi R^{3} \alpha_{\mathrm{C}} Q \sum_{j} q_{j} \frac{\partial n_{j}}{\partial R} .
\end{gathered}
$$

The total pressure, entropy, and chemical potential of a strangelet then becomes

$$
\begin{gathered}
P_{\text {total }}=P+P_{\mathrm{C}}, \\
S_{\text {total }}=S+S_{\mathrm{C}}, \\
\mu_{i, \text { total }}=\mu_{i}^{*}+\frac{1}{3} \frac{\partial m_{i}}{\partial n_{\mathrm{b}}} \frac{\partial \Omega_{0}}{\partial m_{i}}+\mu_{i, \mathrm{C}} .
\end{gathered}
$$

\section{DENSITY AND/OR TEMPERATURE DEPENDENT PARTICLE MASSES}

The equivalent quark masses are usually parametrized as

$$
m_{i}=m_{i 0}+m_{\mathrm{I}}
$$

where $m_{\mathrm{I}}$ accounts for the strong interaction, and $m_{i 0}$ the current quark mass with $m_{u 0}=5 \mathrm{MeV}, m_{d 0}=10 \mathrm{MeV}$, and $m_{s 0}=100 \mathrm{MeV}$.

According to MIT bag model, the quark mass scaling for baryon densitydependent quark mass model was initially parametrized as [50-53]

$$
m_{\mathrm{I}}=\frac{B}{3 n_{\mathrm{b}}},
$$

where $B$ is the bag constant. Based on the in-medium chiral condensates and linear confinement condition, a new quark mass scaling was later derived [54],

$$
m_{\mathrm{I}}=\frac{D}{n_{\mathrm{b}}^{1 / 3}},
$$

where $D$ corresponds to the confinement parameter and is connected to the string tension, chiral symmetry restoration density, and chiral condensate in vacuum. The one-gluonexchange interaction can be considered as well by adding a new term [55], i.e.,

$$
m_{\mathrm{I}}=\frac{D}{n_{\mathrm{b}}^{1 / 3}}-C n_{\mathrm{b}}^{1 / 3}
$$

where $C$ represents the strength of one-gluon-exchange interaction. By expanding $m_{\mathrm{I}}$ as a Laurent series of Fermi 
momentum, the effects of perturbation interactions can be considered, where a new mass scaling was formulated as [8]

$$
m_{\mathrm{I}}=\frac{D}{n_{\mathrm{b}}^{1 / 3}}+C n_{\mathrm{b}}^{1 / 3}
$$

Here, $C$ takes positive values and represents the strength of a first-order perturbation interaction. In order to describe the deconfinement phase transition, this formula is extended to the cases of finite temperature [56], i.e.,

$$
m_{\mathrm{I}}=\frac{D}{n_{\mathrm{b}}^{1 / 3}}\left(1+\frac{8 T}{\Lambda} e^{-\Lambda / T}\right)^{-1}+C n_{b}^{1 / 3}\left(1+\frac{8 T}{\Lambda} e^{-\Lambda / T}\right),
$$

where the constant $\Lambda=280 \mathrm{MeV}$.

In addition, we need to know the equivalent mass of gluons since their contributions cannot be neglected. The gluon mass has been described according to a fast convergent expression of the QCD coupling base on the recent lattice data [57]. Borsányi et al. provided 48 values of the pressure by the lattice simulations, where we use the least square method to get the most effective fitting result [57]. Here, we define the scaled temperature as $x=T / T_{c}$, where $T_{c}$ is the critical temperature. For $T<T_{c}$, the expression of gluon's equivalent mass is

$$
\frac{m_{g}}{T}=\sum_{i} a_{i} x^{i}=a_{0}+a_{1} x+a_{2} x^{2}+a_{3} x^{3},
$$

with $a_{0}=67.018, a_{1}=-189.089, a_{2}=212.666, a_{3}=$ -83.605 . For $T>T_{c}$, the expression of the gluon's equivalent mass is

$$
\frac{m_{g}}{T}=\sum_{i} b_{i} \alpha^{i}=b_{0}+b_{1} \alpha+b_{2} \alpha^{2}+b_{3} \alpha^{3},
$$

where $b_{0}=0.218, b_{1}=3.734, b_{2}=-1.160, b_{3}=0.274$. The QCD coupling constant $\alpha=\alpha_{s} / \pi=g^{2} /\left(4 \pi^{2}\right)$ depends on the renormalization scheme and runs according to the renormalization equation. The expansion equation of $\alpha$ can be obtained by solving the renormalization group equation [58], which gives

$$
\alpha=\frac{\beta_{0}}{\beta_{0}^{2} \ln (u / \Lambda)+\beta_{1} \ln \ln (u / \Lambda)} .
$$

Here, $\beta_{0}=11 / 2-N_{f} / 3, \beta_{1}=51 / 4-19 N_{f} / 12, u / \Lambda=$ $\sum_{i} c_{i} x^{i}=c_{0}+c_{1} x, c_{0}=1.054, c_{1}=0.479$.

\section{PROPERTIES OF STRANGELETS}

To study the properties of strangelets, the finite size effects need to be considered, which are treated with the multiple reflection expansion (MRE) method [9,24-26]. The MRE method introduces a modification to the density of states, which is given by

$\rho_{i}\left(p, R, m_{i}\right)=\frac{d_{i} p^{2}}{2 \pi^{2} V}\left[V+\frac{S_{r}}{p} f_{S}\left(\frac{m_{i}}{p}\right)+\frac{C_{r}}{p^{2}} f_{C}\left(\frac{m_{i}}{p}\right)\right]$.

The index $i$ represents the particle type, while $V, S_{r}$, and $C_{r}$ are the volume, surface area [9], and curvature term [24-26] of a strangelet. For a spherical system, $V=4 \pi R^{3} / 3$, $S_{r}=4 \pi R^{2}, C_{r}=8 \pi R$. The degeneracy factors are $d_{q}=$ 3 (colors) $\times 2$ (spins) $=6$ for quarks $(q=u, d, s)$ and $d_{g}=$ 8 (colors) $\times 2$ (spins $)=16$ for gluons. The functions $f_{S}$ and $f_{C}$ are given as follows:

$$
\begin{gathered}
f_{S}\left(\frac{m_{i}}{p}\right)=-\frac{1}{2} \arctan \left(\frac{m_{i}}{p}\right), \\
f_{C}\left(\frac{m_{i}}{p}\right)=\frac{1}{6}\left[1-\frac{3 p}{2 m_{i}} \arctan \left(\frac{m_{i}}{p}\right)\right] .
\end{gathered}
$$

According to the baryon densitydependent quark mass model, the thermodynamic potential density of free particles is

$$
\Omega_{0}=\Omega_{0}^{+}+\Omega_{0}^{-}+\Omega_{0}^{g} .
$$

The contributions of gluons $\Omega_{0}^{g}$, quarks $\Omega_{0}^{+}$, and antiquarks $\Omega_{0}^{-}$are given by

$$
\begin{gathered}
\Omega_{0}^{g}=T \int_{0}^{\infty} \ln \left[1-e^{-\sqrt{p^{2}+m_{g}^{2}} / T}\right] \rho_{g} \mathrm{~d} p, \\
\Omega_{0}^{ \pm}=-\sum_{i} \int_{0}^{\infty} T \ln \left[1+e^{-\left(\sqrt{p^{2}+m_{i}^{2}} \mp \mu_{i}^{*}\right) / T}\right] \rho_{i} \mathrm{~d} p .
\end{gathered}
$$

Based on Eqs. (17)-(19), the specific expressions of each thermodynamic quantity at finite temperature can then be obtained. Note that at small radii and momenta the density of state in Eq. (29) becomes negative and is hence unphysical. An infrared cutoff [59] is usually introduced to treat the unphysical contributions in Eqs. (33) and (34), which is not included here since their contributions are small.

We know that there are weak interaction processes $d, s \leftrightarrow u+e+\bar{\nu}_{e}$ and $s+u \leftrightarrow u+d$ in strangelets. Because neutrinos can freely enter and exit SQM and the electron Compton wavelength is much larger than the size of strangelets with $A \ll 10^{7}$, the chemical potentials of neutrinos and electrons are thus zero, which gives

$$
\mu_{u, \text { total }}=\mu_{d, \text { total }}=\mu_{s, \text { total }}
$$


with the chemical potential determined by Eq. (19). For a given baryon number $A$, the baryon number conservation needs to be fulfilled, i.e.,

$$
A=\frac{1}{3} \sum_{i} N_{i}=\frac{1}{3} \sum_{i} n_{i} V
$$

In addition, the pressures of the strangelets must be zero to maintain mechanical stability, i.e.,

$$
P=0,
$$

which is equivalent to minimize the total free energy at fixed $N_{i}$ as usually adopted in the literature $[26,60]$.

Therefore, we could obtain the energy density, particle number density, pressure, entropy, and chemical potential by solving Eqs. (35)-(37). We define the ratios of charge number to baryon number and strangeness quantum number to quark number as

$$
\begin{aligned}
f_{z}=Z / A & =\left(\frac{2}{3} N_{u}-\frac{1}{3} N_{d}-\frac{1}{3} N_{s}\right) / A, \\
f_{s} & =S / \sum_{q} N_{q}=N_{s} / 3 A .
\end{aligned}
$$

In Fig. 1, we present the energy per baryon as a function of the baryon number $A$ at $T=0 \mathrm{MeV}$ and $T=20 \mathrm{MeV}$ for fixed parameters $C$ and $D$ [8]. The solid, dashed, and dotted curves correspond, respectively, to the temperature $T=20,0 \mathrm{MeV}$, and ${ }^{56} \mathrm{Fe}$. The blue, red, and black curves correspond, respectively, to the parameter sets $(C, \sqrt{D}$ in $\mathrm{MeV}):(-0.1,160),(-0.2,164)$, and $(-0.3,168)$. It can be seen from Fig. 1 that the strangelets under three sets of parameters are absolutely stable at zero temperature but not at $T=20 \mathrm{MeV}$. The energy per baryon of strangelets decreases with increasing baryon number $A$. This is consistent with the trend of Jensen and Madsen's results at $T=0$ [61], and the value is slightly larger. It is worth

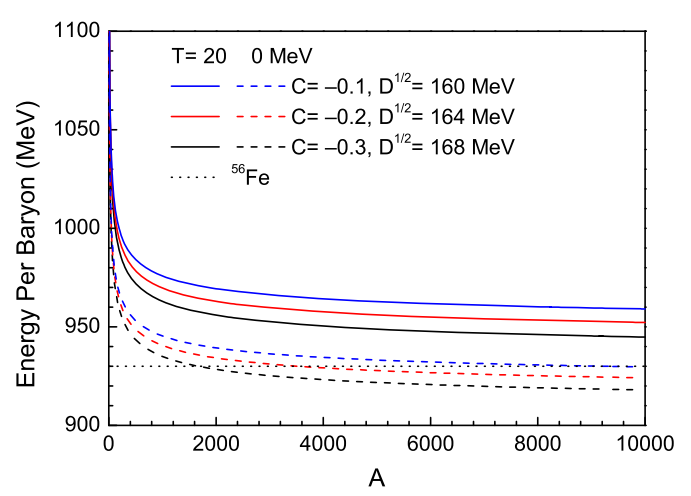

FIG. 1. Energy per baryon of $\beta$-stable strangelets as a function of baryon number.

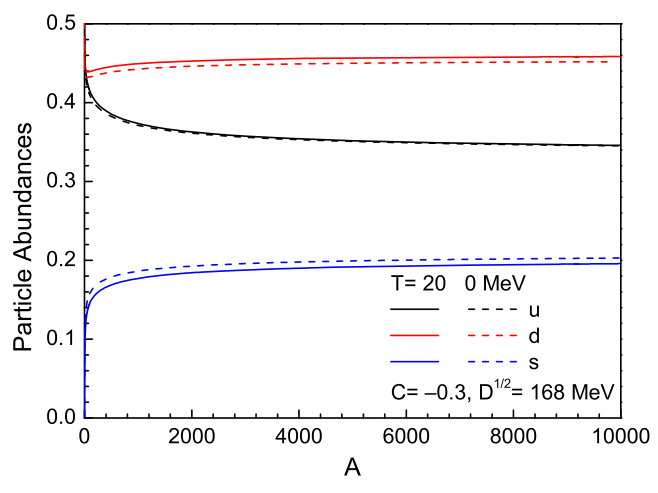

FIG. 2. Particle abundance for $\beta$-stable strangelets as a function of baryon number.

mentioning that strangelets would emit neutrons when $E / A>m_{n} \approx 939 \mathrm{MeV}$ [9]. This suggests that strangelets with large baryon numbers could be absolutely stable at small temperature, which provides the possibility of detecting strangelets from the particles emitted by unknown astrophysical sources where those low temperature and high baryon numbers are expected.

We can see the dependence of the particle abundances of the $u, d$, and $s$ quarks on the baryon number from Fig. 2 . The black, red, and blue curves correspond, respectively, to the $u, d$, and $s$ quarks. With the increase of $A$, the particle abundances of the $u$ quark is decreasing, while those of $d$ and $s$ quarks increase. That is because the number of weak interaction processes $d, s \leftrightarrow u+e+\bar{\nu}_{e}$ and $s+u \leftrightarrow u+$ $d$ in the direction of $s$ increase, accompanying the increase of $A$. Combining with Fig. 1, we find that the stable strangelets only occur in the high strangeness, which is consistent with Zhang and Su's results [62].

In Fig. 3, we show the dependence of the mechanically stable radii of strangelets on the baryon number at $T=0$ and $T=20 \mathrm{MeV}$. The solid and dashed curves correspond, respectively, to the temperature $T=50$ and $0 \mathrm{MeV}$. The blue, red, and black curves correspond, respectively, to the

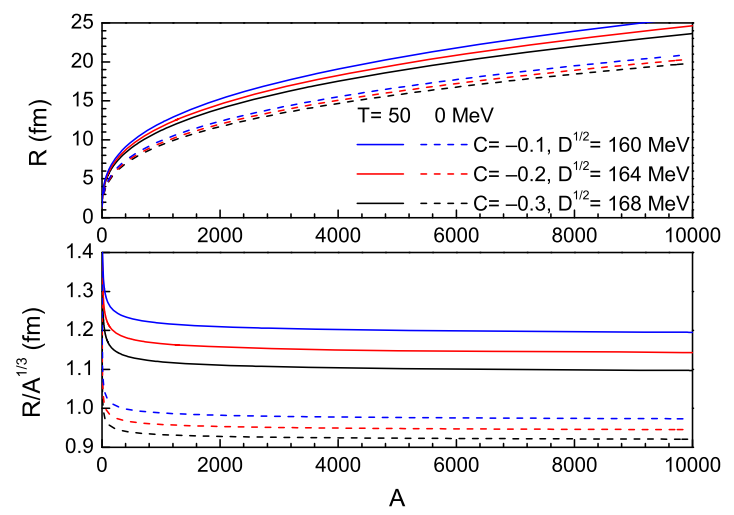

FIG. 3. Mechanically stable radii of $\beta$-stable strangelets as functions of baryon number. 


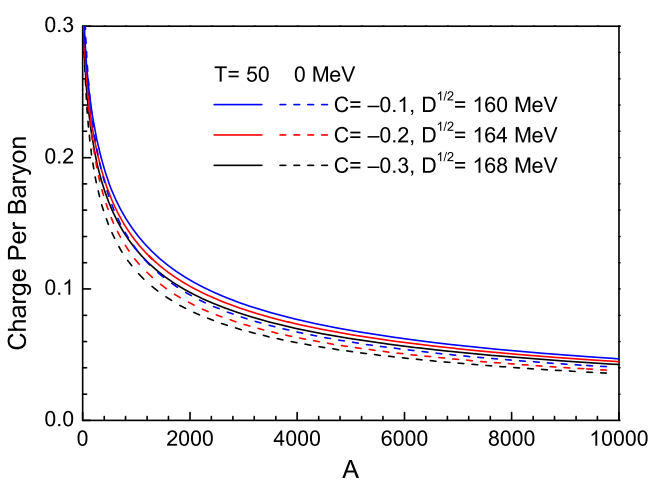

FIG. 4. Charge-to-mass ratios $f_{z}$ of $\beta$-stable strangelets as functions of baryon number.

parameter sets $(C, \sqrt{D}$ in $\mathrm{MeV}):(-0.1,160),(-0.2,164)$, and $(-0.3,168)$. It can be seen from Fig. 3 that the mechanically stable radius $R$ increases with baryon number $A$ while the ratio $R / A^{1 / 3}$ decreases. Moreover, the ratio $R / A^{1 / 3}$ decreases slowly and eventually tends to be a constant as $A \rightarrow \infty$, corresponding to a cube root relation $R=r_{0} A^{1 / 3}$ with a constant $r_{0}$. This is consistent with the conclusion of previous model studies such as the NJL model [63].

The dependence of the charge-to-mass ratio $f_{z}$ of the strangelets on the baryon number is depicted in Fig. 4 at $T=0 \mathrm{MeV}$ and $T=50 \mathrm{MeV}$. It can be seen that $f_{z}$ decreases with baryon number $A$ and tends to zero when the baryon number $A$ is large. This is consistent with the prediction of electrically neutral SQM and the result in Fig. 2. Note that at $f_{z} \rightarrow 0$, we do not consider the contribution of Coulomb interactions. Furthermore, we notice that the one-gluon-exchange interaction reduces $f_{z}$. Note that a possible candidate of a strangelet with mass $A>1000$ and charge 46 was reported from cosmic ray experiments [64].

We use the ratio of the strange quark number to total quark number $f_{s}=N_{s} / 3 \mathrm{~A}$ to express the strangeness of

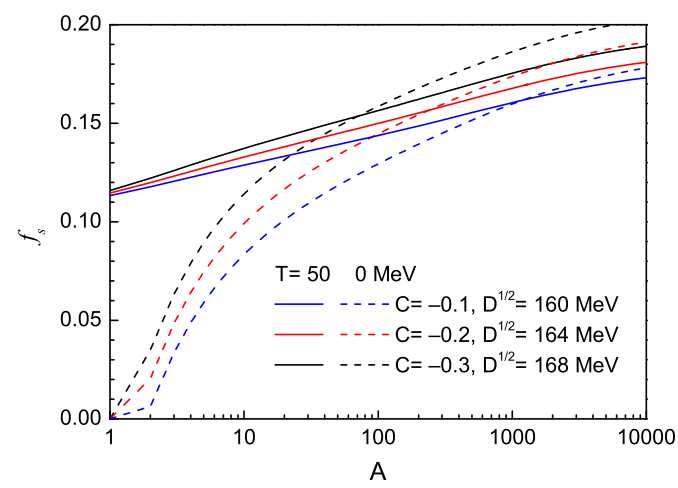

FIG. 5. Ratio of the strange quark number to total quark baryon number $f_{s}$ as a function of baryon number.

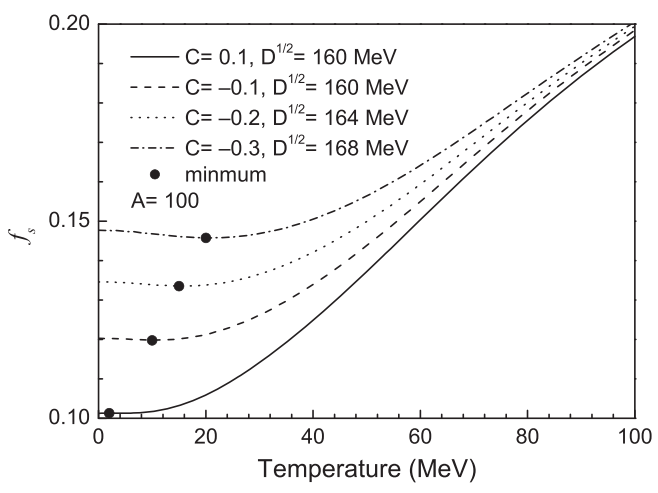

FIG. 6. Ratio of the strange quark number to total quark baryon number $f_{s}$ as a function of temperature when $A$ is 100 .

strangelets, which is shown in Fig. 5. It can be seen that $f_{s}$ is increasing and tends to a stable value when the baryon number $A$ is large. Furthermore, for strangelets with small $A$, we find that the strangeness gets larger for higher temperatures, while the trend is reversed at large $A$. The strangeness of strangelets tends to a constant value at any temperature. There are some interesting points, which we explain in combination with Fig. 6.

In Fig. 6, we can see a minimum point of the strangeness of strangelets for fixed parameters $C$ and $D$ at $T=T_{0}$. The solid, dash, dotted, and dashed dotted curves correspond, respectively, to the parameter sets $(C, \sqrt{D}):(0.1,160)$, $(-0.1,160),(-0.2,164)$, and $(-0.3,168)$. It is found that the one-gluon-exchange interaction increases $f_{s}$ and $T_{0}$, while the first-order perturbation interaction reduces $f_{s}$ and eventually $T_{0}$ reaches zero.

Next, we study the relationship between the temperature and energy per baryon in Fig. 7. We choose the parameter sets $(C, \sqrt{D}):(0.1,160),(-0.1,160),(-0.2,164)$, and $(-0.3,168)$. We can see that the higher the temperature is, the great the energy per baryon is at $A=100$ due to the increment of the thermal energy of quarks and gluons. Therefore, strangelets will become unstable and are not

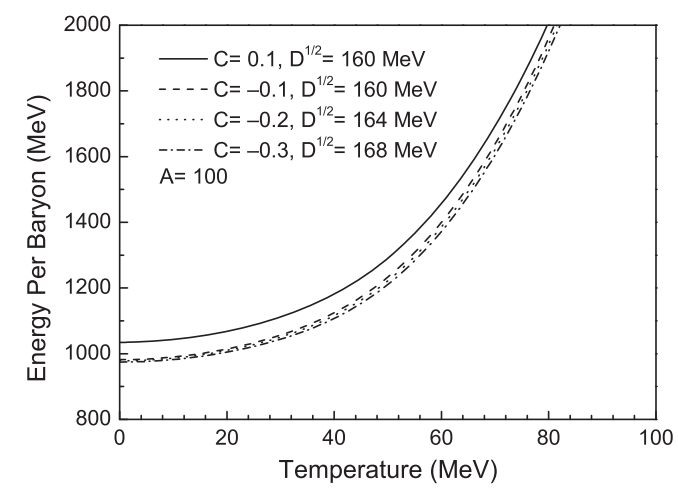

FIG. 7. Energy per baryon as a function of temperature when $A$ is 100 . 


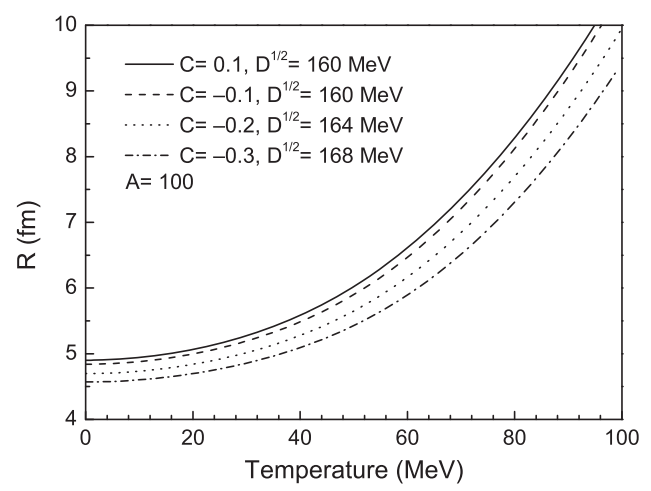

FIG. 8. Mechanically stable radius as a function of temperature when $A$ is 100 .

likely to exist. In addition, it is found that the one-gluonexchange interaction reduces the energy, and the perturbative interaction increases the energy.

In Fig. 8, we note that the mechanically stable radius is a monotone increasing function of the temperature when $A$ is 100. Furthermore, we notice that there should be an exponential function relation between $R$ and $T$. This is consistent with the result of the energy per baryon in Fig. 7. We also find that the perturbative interaction increases the mechanically stable radius while the one-gluon-exchange interaction decreases the mechanically stable radius at fixed $D$.

In Fig. 9, we present the charge to mass ratio as a function of temperature when $A$ is 100 . We see that $f_{z}$ does not decrease monotonically with temperature, where a maximum value exists for the charge to mass ratios of strangelets. Let us say the temperature at this point is $T_{\max }$. At $T<T_{\max }$, the higher the temperature is, the larger the strangeness of strangelets is, while otherwise the opposite at $T>T_{\max }$. Note that the different parameter sets $(C, \sqrt{D})$ have different maximum points. Furthermore, we see that the first-order perturbative interaction increases $f_{z}$ and reduces $T_{\max }$, and the one-gluon-exchange interaction

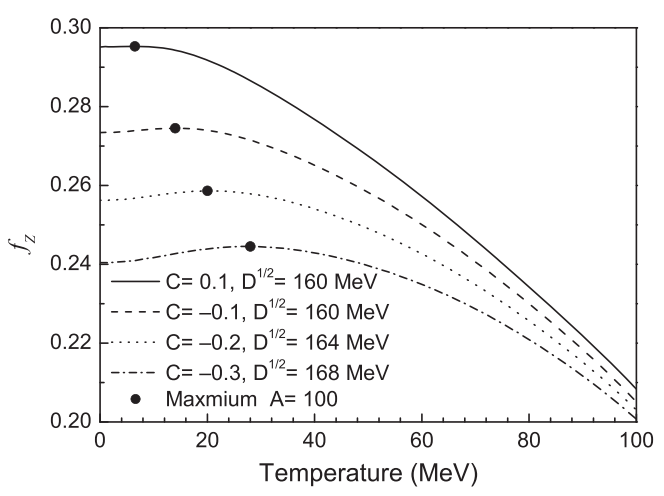

FIG. 9. The ratio of charge number to the baryon number $f_{z}$ as a function of temperature when $A$ is 100 .

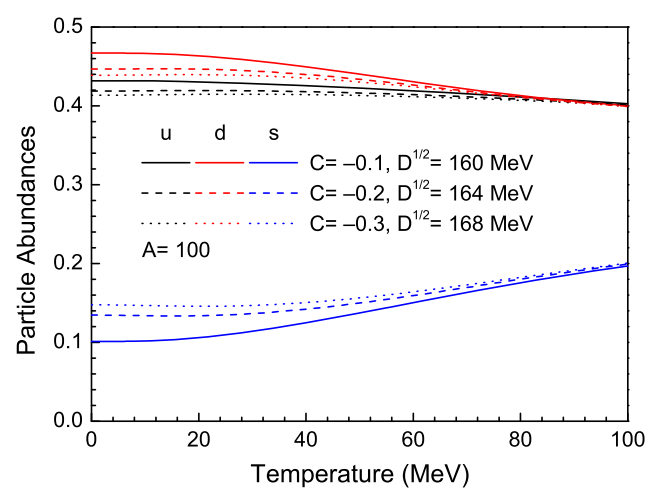

FIG. 10. Particle abundances for $\beta$-stable strangelets as functions of temperature when $A$ is 100 .

decreases $f_{z}$ and increases $T_{\max }$. This is consistent with the result of $f_{s}$ in Fig. 6.

The dependence of the particle abundances of quarks on the temperature is depicted in Fig. 10. The black, red, and blue curves correspond, respectively, to the quarks $u, d$, and $s$. The solid, dashed, dotted, and dashed dotted curves correspond, respectively, to the parameter sets $(C, \sqrt{D})$ : $(0.1,160),(-0.1,160),(-0.2,164)$, and $(-0.3,168)$. With the increase of temperature, the particle abundances of $u$ and $d$ quarks are decreasing, while that of the $s$ quark increases. This is because $u$ and $d$ quarks are converted to $s$ quarks at high temperature. We also find that the first-order perturbation interaction increases the particle abundances of $u$ and $d$ quarks and the one-gluon-exchange interaction decreases the particle abundances of $u$ and $d$ quarks. At the same time, the first-order perturbation interaction decreases the particle abundances of $s$ quarks, and the one-gluonexchange interaction increases the particle abundances of $s$ quarks.

Figure 11 shows the entropy per baryon as a function of temperature for the different parameter sets $(C, \sqrt{D})$. The first-order perturbation interaction improves the entropy, and the one-gluon-exchange interaction reduces the entropy. It is an increasing function of temperature

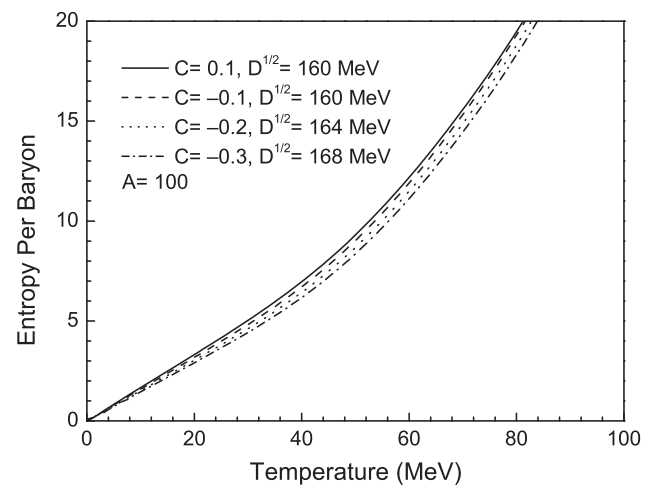

FIG. 11. Entropy per baryon as a function of the temperature for fixed parameter sets $(C, \sqrt{D})$ when $A$ is 100 . 
and approaches zero at vanishing temperature. This is guaranteed by

$$
\lim _{T \rightarrow 0} \partial m_{q} / \partial T=0
$$

\section{SUMMARY}

We have investigated the properties of strangelets at finite temperature by the baryon densitydependent quark mass model with a quark mass scaling with confinement and perturbative interaction. We have analyzed the contribution of Coulomb interaction by a thermodynamic self-consistent way and obtained the contributions to the pressure and chemical potential. Based on the baryon densitydependent quark mass model with self-consistent thermodynamic treatment, we studied the properties of strangelets, such as the energy per baryon, mechanically stable radius, strangeness, charge property, and particle abundances. It is found that the energy per baryon and $f_{z}$ decreased and the mechanically stable radius and strangeness increased as baryon number $A$ increases. In addition, the obtained energy per baryon, mechanically stable radius, and strangeness increased with temperature, and $f_{z}$ decreased. These coincide with previous observations for the general behavior of strangelets as we vary the baryon number and/or temperature [61-63]. For fixed baryon numbers, the strangeness per baryon $f_{s}$ as a function of temperature has a local minimum, while that of charge-to-mass ratio has a local maximum.

\section{ACKNOWLEDGMENTS}

The authors are thankful for the support from the National Natural Science Foundation of China (Grants No. 11875052, No. 11575190, and No. 11135011).
[1] A. R. Bodmer, Phys. Rev. D 4, 1601 (1971).

[2] E. Witten, Phys. Rev. D 30, 272 (1984).

[3] E. Farhi and R. L. Jaffe, Phys. Rev. D 30, 2379 (1984).

[4] G. X. Peng, H. C. Chiang, P. Z. Ning, and B. S. Zhou, Phys. Rev. C 59, 3452 (1999).

[5] G. X. Peng, H. C. Chiang, B. S. Zou, P. Z. Ning, and S. J. Luo, Phys. Rev. C 62, 025801 (2000).

[6] J. F. Xu, G. X. Peng, F. Liu, D. F. Hou, and L. W. Chen, Phys. Rev. D 92, 025025 (2015).

[7] J. F. Xu, Y. A. Luo, L. Li, and G. X. Peng, Phys. Rev. D 96, 063016 (2017).

[8] C. J. Xia, G. X. Peng, S. W. Chen, Z. Y. Lu, and J. F. Xu, Phys. Rev. D 89, 105027 (2014).

[9] M. S. Berger and R. L. Jaffe, Phys. Rev. C 35, 213 (1987).

[10] G. X. Peng, X. J. Wen, and Y. D. Chen, Phys. Lett. B 633, 314 (2006).

[11] S. Banerjee, S. K. Ghosh, S. Raha, and D. Syam, J. Phys. G 25, L15 (1999).

[12] S. Banerjee, S. K. Ghosh, S. Raha, and D. Syam, Phys. Rev. Lett. 85, 1384 (2000).

[13] J. Sandweiss, J. Phys. G 30, S51 (2004).

[14] T. Saito, Y. Hatano, Y. Fukada, and H. Oda, Phys. Rev. Lett. 65, 2094 (1990).

[15] J. Madsen and J. M. Larsen, Phys. Rev. Lett. 90, 121102 (2003).

[16] J. Madsen, Phys. Rev. D 71, 014026 (2005).

[17] M. Merafina, F. G. Saturni, C. Curceanu, R. Del Grande, and K. Piscicchia, Phys. Rev. D 102, 083015 (2020).

[18] G. Appelquist et al., Phys. Rev. Lett. 76, 3907 (1996).

[19] A. Rusek, Phys. Rev. C 54, R15 (1996).

[20] B. V. Gene, J. Phys. G 25, 411 (1999).

[21] M. W. Richard, Int. J. Mod. Phys. E 15, 37 (2006).

[22] C. Greiner, P. Koch, and H. Stöcker, Phys. Rev. Lett. 58, 1825 (1987).
[23] C. Greiner and H. Stöcker, Phys. Rev. D 44, 3517 (1991).

[24] J. Madsen, Phys. Rev. Lett. 70, 391 (1993).

[25] J. Madsen, Phys. Rev. D 47, 5156 (1993).

[26] J. Madsen, Phys. Rev. D 50, 3328 (1994).

[27] M. G. Mustafaand and A. Ansari, Phys. Rev. D 53, 5136 (1996).

[28] Y. Zhang and R. K. Su, Phys. Rev. C 67, 015202 (2003).

[29] M. G. Alford, K. Rajagopal, S. Reddy, and A. W. Steiner, Phys. Rev. D 73, 114016 (2006).

[30] G. Lugones and A. G. Grunfeld, Phys. Rev. C 103, 035813 (2021).

[31] G. Lugones and A. G. Grunfeld, Phys. Rev. D 104, L101301 (2021).

[32] E. S. Fraga and P. Romatschke, Phys. Rev. D 71, 105014 (2005).

[33] Y. Shi and R. X. Xu, Astrophys. J 596, L75 (2003).

[34] R. Xu, Int. J. Mod. Phys. D 19, 1437 (2010).

[35] S. Plumari, G. F. Burgio, V. Greco, and D. Zappala, Phys. Rev. D 88, 083005 (2013).

[36] G. S. Khadekar and Rupali Wanjari, Int. J. Theor. Phys. 51, 1408 (2012).

[37] X. J. Wen, Phys. Rev. D 88, 034031 (2013).

[38] A. A. Isayev and J. J. Yang, J. Phys. G 40, 035105 (2013).

[39] X. Y. Wang and I. A. Shovkovy, Phys. Rev. D 82, 085007 (2010).

[40] M. Huang and I. A. Shovkovy, Phys. Rev. D 70, 051501(R) (2004).

[41] T. Bao, G. Z. Liu, E. G. Zhao, and M. F. Zhu, Eur. Phys. J. A 38, 287 (2008).

[42] J. X. Hou, G. X. Peng, C. J. Xia, and J. F. Xu, Chin. Phys. C 39, 015101 (2015).

[43] S. Yasui, A. Hosaka, and H. Toki, Phys. Rev. D 71, 074009 (2005). 
[44] X. J. Wen, J. Y. Li, J. Q. Liang, and G. X. Peng, Phys. Rev. C 82, 025809 (2010).

[45] G. X. Peng, A. Li, and U. Lombardo, Phys. Rev. C 77, 065807 (2008).

[46] G. X. Peng, S. W. Chen, Q. Chang, and H. H. Tang, Int. J. Mod. Phys. E 19, 1837 (2010).

[47] S. Chakrabarty, S. Raha, and B. Sinha, Phys. Lett. B 229 , 112 (1989).

[48] O. G. Benvenuto and G. Lugones, Phys. Rev. D 51, 1989 (1995).

[49] X. J. Wen, X. H. Zhong, G. X. Peng, P. N. Shen, and P. Z. Ning, Phys. Rev. C 72, 015204 (2005).

[50] G. N. Fowler, S. Raha, and R. M. Weiner, Z. Phys. C 9, 271 (1981).

[51] S. Chakrabarty, S. Raha, and B. Sinha, Phys. Lett. B 229, 112 (1989).

[52] S. Chakrabarty, Phys. Rev. D 43, 627 (1991).

[53] S. Chakrabarty, Phys. Rev. D 48, 1409 (1993).
[54] G. X. Peng, H. C. Chiang, J. J. Yang, L. Li, and B. Liu, Phys. Rev. C 61, 015201 (1999).

[55] S. W. Chen, L. Gao, and G. X. Peng, Chin. Phys. C 36, 947 (2012).

[56] Z. Y. Lu, G. X. Peng, S. P. Zhang, M. Ruggieri, and V. Greco, Nucl. Sci. Tech. 27, 145 (2016).

[57] Sz. Borsányi, G. Endrődi, Z. Fodor, S. D. Katz, and K. K. Szabo, J. High Energy Phys. 01 (2012) 138.

[58] G. X. Peng, Phys. Lett. B 634, 413 (2006).

[59] G. Neergaard and J. Madsen, Phys. Rev. D 60, 054011 (1999).

[60] G. Lugones and A. G. Grunfeld, Phys. Rev. D 84, 085003 (2011).

[61] D. M. Jensen and J. Madsen, Phys. Rev. D 53, R4719 (1996).

[62] Y. Zhang and R. K. Su, Phys. Rev. C 67, 015202 (2003).

[63] O. Kiriyama, Phys. Rev. D 72, 054009 (2005).

[64] S. Banerjee, S. K. Ghosh, S. Raha, and D. Syam, Phys. Rev. Lett. 85, 1384 (2000). 